\title{
Role of the Primary Motor and Dorsal Premotor Cortices in the Anticipation of Forces during Object Lifting
}

\author{
Philippe A. Chouinard, ${ }^{1}$ Gabriel Leonard, ${ }^{1}$ and Tomáš Paus ${ }^{1,2}$ \\ ${ }^{1}$ Cognitive Neuroscience Unit, Montreal Neurological Institute, McGill University, Montreal, Quebec H3A 2B4, Canada, and 2Brain \& Body Centre, \\ University of Nottingham, Nottingham NG7 2RD, United Kingdom
}

\begin{abstract}
When lifting small objects, people apply forces that match the expected weight of the object. This expectation relies in part on information acquired during a previous lift and on associating a certain weight with a particular object. Our study examined the role of the primary motor and dorsal premotor cortices in predicting weight based either on information acquired during a previous lift (no-cue experiment) or on arbitrary color cues associated with a particular weight (cue experiment). In the two experiments, subjects used precision grip to lift two different weights in a series of trials both before and after we applied low-frequency repetitive transcranial magnetic stimulation over the primary motor and dorsal premotor cortices. In the no-cue experiment, subjects did not receive any previous information about which of two weights they would have to lift. In the cue experiment, a color cue provided information about which of the two weights subjects would have to lift. Our results demonstrate a double dissociation in the effects induced by repetitive stimulation. When applied over the primary motor cortex, repetitive stimulation disrupted the scaling of forces based on information acquired during a previous lift. In contrast, when applied over the dorsal premotor cortex, repetitive stimulation disrupted the scaling of forces based on arbitrary color cues. We conclude that the primary motor and dorsal premotor cortices have unique roles during the anticipatory scaling of forces associated with the lifting of different weights.
\end{abstract}

Key words: repetitive transcranial magnetic stimulation; dorsal premotor area; primary motor area; weight prediction; precision grip; visuomotor association

\section{Introduction}

The precision grip has been investigated extensively in humans (Johansson, 1996). People typically use the tips of the index finger and thumb when lifting small objects. The lifting of such objects requires fine motor control; too much force can damage the object or result in an excessive lifting movement, and too little force can cause the object to slip away. Throughout life, we build internal representations for the weight of different objects (Gordon et al., 1993; Wolpert and Flanagan, 2001). This provides us with the ability to apply forces for lifting objects using feedforward mechanisms. In cases when the weight is lighter than expected, somatosensory information related to lift-off will generate corrective forces to stabilize the object (Johansson and Westling, 1988). In cases when the weight is heavier than expected, the absence of an expected lift-off will generate corrective forces to overcome gravity on the object (Johansson and Westling, 1988).

Information acquired by a recent lift can influence the anticipatory scaling of forces for a subsequent lift (Johansson and Westling, 1988; Gordon et al., 1993; Fellows et al., 1998). When the weight of an object changes unexpectedly without any

Received Aug. 13, 2004; revised Jan. 13, 2005; accepted Jan. 14, 2005.

This work was supported by the Canadian Institute of Health Research (MT-14505) and by the Canadian Foundation for Innovation. We thank Dr. Valeria Della-Maggiore, Oriane Landry, and the anonymous reviewers for their thoughtful comments on this manuscript, Dr. Catherine Poulsen for statistical advice, and Jocelyn Roy for constructing the precision grip apparatus.

Correspondence should be addressed to Tomáš Paus, Brain \& Body Centre, University of Nottingham, University Park, Nottingham NG7 2RD, UK. E-mail: tomas.paus@nottingham.ac.uk.

D0I:10.1523/JNEUROSCI.4649-04.2005

Copyright $\odot 2005$ Society for Neuroscience $\quad$ 0270-6474/05/252277-08\$15.00/0 changes in appearance, people will generate inappropriate forces on the first lift and quite accurate forces on the subsequent lift. This adaptation indicates that the motor system can update quickly information pertaining to the properties of an object and is thought to involve processes similar to those used to correct for errors made in predicting weight (Johansson and Westling, 1988). Cell recording studies in the monkey demonstrate that a population of primary motor neurons processes information related to a recent experience by altering their firing properties during motor adaptation ( $\mathrm{Li}$ et al., 2001). We predict that repetitive transcranial magnetic stimulation (rTMS) applied over the primary motor cortex can disrupt the scaling of forces based on information acquired during a previous lift.

The dorsal premotor cortex selects movements mainly on the basis of learned associations (Wise and Murray, 2000). People generate more accurate forces during the lifting of small objects after they have learned to associate arbitrary color cues with weight (Cole and Rotella, 2002). Semantic identification can also influence forces applied during the lifting of commonly used objects (Gordon et al., 1993). Rapid associative learning is thought to generate internal representations that link object identification with the scaling of forces required to lift them. People can learn new associations as quickly as by the second trial and reproduce forces accurately for up to $24 \mathrm{~h}$ (Gordon et al., 1991b; Flanagan et al., 2001). Lesion studies in the monkey have shown that the removal of the dorsal premotor cortex disrupts the ability to use arbitrary visual cues to make or withhold particular movements (Halsband and Passingham, 1982, 1985; Pet- 
rides, 1982, 1985a). We predict that rTMS applied over the dorsal premotor cortex can disrupt the scaling of forces based on arbitrary color cues.

\section{Materials and Methods}

Overview. Two groups of subjects participated in each of two experiments, namely the no-cue and cue experiments; the individual volunteers were assigned to the two experiments at random. We applied $15 \mathrm{~min}$ of $1 \mathrm{~Hz}$ rTMS over the left primary motor cortex on one day and the left dorsal premotor cortex on another day. We counterbalanced the order in which repetitive stimulation was delivered over the two brain sites in a given subject. Figure $1 A$ illustrates the time course for one session. We acquired motor-evoked potentials (MEPs) by delivering single-pulse TMS over the left primary motor cortex $22-20,12-10$, and 2-0 min before the onset of rTMS and 0-2, 10-12, 20-22, and 30-32 min after the conclusion of rTMS. We also acquired precision grip measurements 20-12 and 10-2 min before the onset of rTMS and 12-20 and 22-30 min after the conclusion of rTMS.

Participants. Subjects in the two experiments matched for sex, age, handedness, pinch strength, and manual dexterity. Four female and four male subjects (19-30 years of age; mean \pm SEM, $24.4 \pm 1.4$ ) participated in the no-cue experiment, and another four female and four male subjects (21-36 years of age; mean \pm SEM, $25.6 \pm 1.6)$ participated in the cue experiment. All subjects had a strong right-hand preference as determined by a handedness questionnaire (Crovitz and Zener, 1965); a paired $t$ test revealed no significant group difference in handedness $(p=$ 0.73 ). We tested pinch strength for both hands using a Jamar pinch dynamometer (Sammons Preston, Bolinbrook, IL) and manual dexterity for both hands using the grooved pegboard test, model 32025 (Lafayette Instrument Company, Lafayette, IN). Paired $t$ tests revealed no significant group differences in pinch strength for either the right hand $(p=$ 0.27 ) or the left hand ( $p=0.29)$ and in performance times in the grooved pegboard test for either the right hand $(p=0.80)$ or the left hand ( $p=$ 0.78 ). All subjects provided informed written consent before participation. The Research Ethics Board of the Montreal Neurological Institute and Hospital approved all experimental procedures. We selected subjects for whom we had previously acquired anatomical magnetic resonance images (MRIs) [160-192 contiguous 1-mm-thick sagittal slices; Siemens AG (Erlangen, Germany) Vision 1.5-T system] and who had low resting motor thresholds. We established the latter criterion to prevent overheating of the stimulating coil.

Apparatus for precision grip. We constructed a manipulandum (Fig. $1 B$ ) based on the classical apparatus used by Johansson and Westling (1984). The contact surface with which subjects grasped the manipulandum consisted of sandpaper (no. 150) attached to both sides of the handle. We measured the grip force using a set of strain-gauge transducers attached to the handle where subjects grasped with the index finger and the load force using a set of strain gauges attached to the base of the manipulandum. The resulting signals fed continuously to a Grass model 15A54 quad amplifier (Astro-Med, West Warwick, RI) at a sampling rate of $800 \mathrm{~Hz}$. We saved all data on a laptop computer for off-line analysis. We also attached to the base of the manipulandum an aluminum rod that passed through a hole in the table that held a weight carrier at its bottom end. This allowed us to add or remove from the carrier a $200 \mathrm{~g}$ weight without the subject seeing us change weights. Subjects wore earphones and listened to white noise at an intensity that they could tolerate comfortably ( $\sim 60$ to $\sim 80 \mathrm{~dB}$ ). Our pilot experiments revealed that the white noise helped prevent subjects from hearing us add or remove the weight and thus realize when a switch in weight occurred between lifts.

Apparatus for transcranial magnetic stimulation. We performed TMS using a Cadwell (Kennewick, WA) high-speed magnetic stimulator and a Cadwell figure-of-eight stimulating coil with a built-in cooling system (Corticoil; two tear-shaped coils of $\sim 5 \mathrm{~cm}$ diameter each). We chose this coil because it produces a magnetic-field maximum of sufficiently small width to allow stimulation of the dorsal premotor cortex without encroaching on the primary motor cortex. A similar coil was previously found to stimulate an estimated volume of $20 \times 20 \times 10 \mathrm{~mm}$ (Cohen et al., 1990; Maccabee et al., 1990; Wassermann et al., 1996). Subjects used

\section{Experimental Set-up}

\section{A}
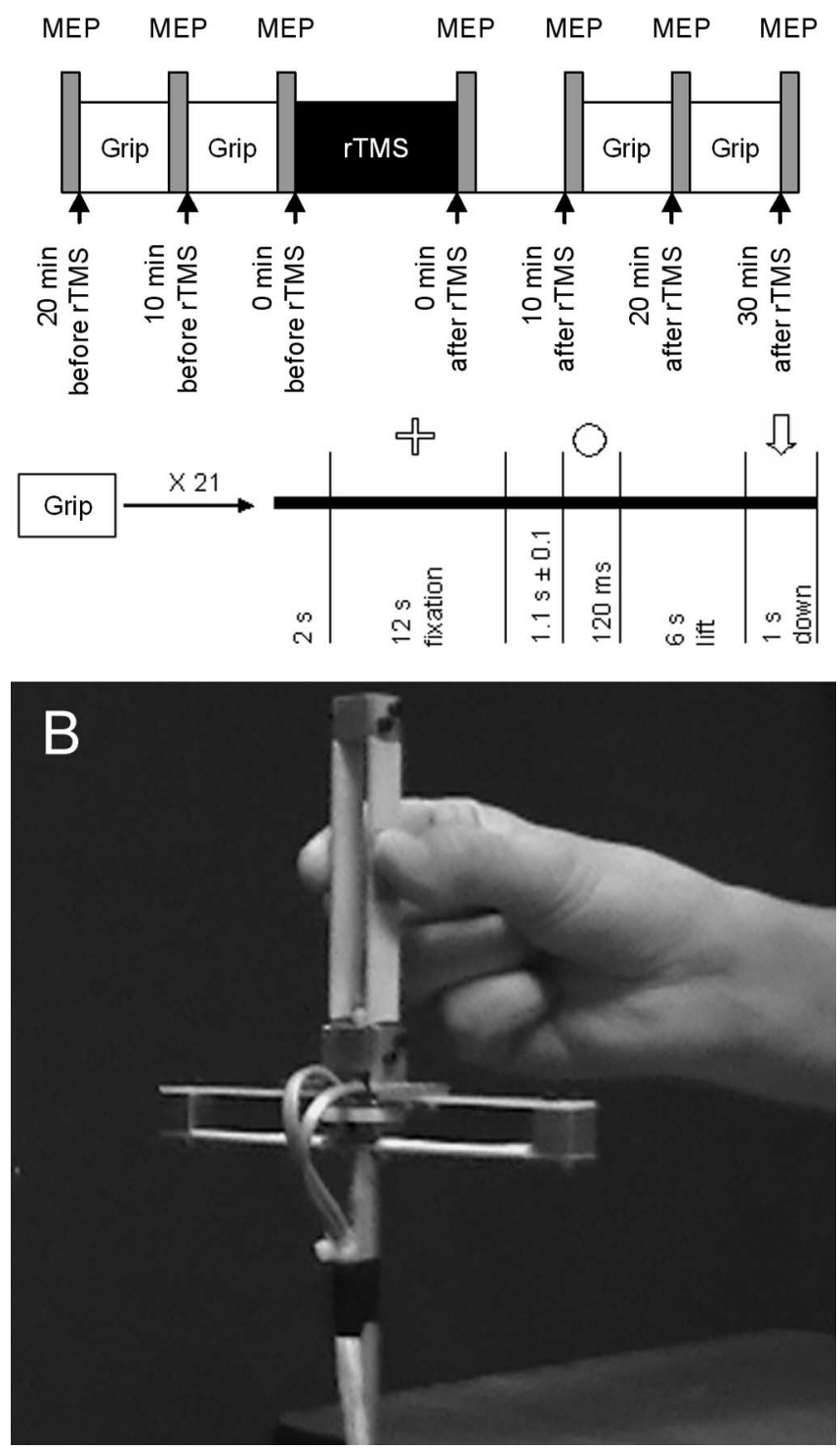

Figure 1. Experimental setup. $A$ illustrates the chronological order of a session. During task performance, subjects performed 21 lifts in which they fixated their gaze on the computer screen until they saw a cue. After cue presentation, they then grasped the manipulandum between the tips of the index finger and thumb and lifted it vertically for a distance of $\sim 10 \mathrm{~cm}$. They maintained the manipulandum in this position until they saw an arrow pointing down on the computer screen. $\boldsymbol{B}$ illustrates the manipulandum that we used to measure precision grip.

a bite bar during stimulation while a mechanical arm held the coil over the target locations. We determined motor thresholds for the relaxed right first dorsal interosseus muscle before each session (Chouinard et al., 2003).

Apparatus for electromyography. We recorded MEPs from the right first dorsal interosseus muscle using $\mathrm{Ag} / \mathrm{AgCl}$ surface electrodes fixed on the skin with a belly-tendon montage. We sampled the electromyographic signal using the Grass amplifier with a bandwidth set at 0.1-3000 $\mathrm{Hz}$ and the sampling rate set at $2000 \mathrm{~Hz}$. We then saved these data on a laptop computer for off-line analysis. We measured the peak-to-peak amplitudes for each MEP using the program Matlab (MathWorks, Natick, MA) and then calculated the mean MEP amplitude for each condition based on the 20 trials. 
Procedures for precision grip. We performed the two experiments in a quiet room with the lights dimmed in which subjects sat comfortably in front of a computer screen. In the no-cue experiment, we presented a white circle as a neutral stimulus before subjects lifted weights of $325 \mathrm{~g}$ (light) or $525 \mathrm{~g}$ (heavy); this circle provided no information about what weight would be lifted. In the cue experiment, we presented a pink circle before subjects lifted a weight of $325 \mathrm{~g}$ and a blue circle before subjects lifted a weight of $525 \mathrm{~g}$. During task performance, subjects performed 21 lifts in which they fixated their gaze on the computer screen until they saw a cue. After cue presentation, they then grasped the manipulandum between the tips of the index finger and thumb and lifted it vertically for a distance of $\sim 10 \mathrm{~cm}$. They maintained the manipulandum in this position until they saw on the computer screen an arrow pointing down.

In the beginning of the first session, we demonstrated how to perform the task properly and then provided subjects with a 5 min training period in which they performed a series of trials with the $325 \mathrm{~g}$ weight. We instructed subjects to grasp the manipulandum between the tips of the index finger and thumb and lift the manipulandum using appropriate forces. We also instructed subjects to lift vertically for a distance of $\sim 10$ $\mathrm{cm}$; the lifting movement of the task required mainly a flexion of the elbow. During the training period, we provided verbal feedback so as to ensure that they grasped the manipulandum with the tips of the index finger and thumb only. We did not provide any additional feedback after this 5 min training period. Figure $1 A$ illustrates the time course of each trial. Subjects performed a total of 21 lifts per block, so that after removing the first trial, we obtained five trials for each of the following four conditions: light-after-light, light-after-heavy, heavy-after-heavy, and heavy-after-light. We predetermined the order of these conditions pseudorandomly and presented a different order for each of the different blocks.

Procedures for transcranial magnetic stimulation. We reduced excitability by applying rTMS over the left primary motor cortex on one day and the left dorsal premotor cortex on another day (Touge et al., 2001; Munchau et al., 2002). Direct corticocortical connections between the dorsal premotor and primary motor cortices are thought to mediate reductions in motor excitability after repetitive stimulation over the dorsal premotor cortex (Munchau et al., 2002). We used single-pulse TMS over the left primary motor cortex to measure MEPs as an index of the effectiveness of rTMS applied over the two sites (Chouinard et al., 2003). We introduced an $\sim 10$ min delay before subjects performed the precision grip task again because we had found previously that it took $\sim 10$ min after repetitive stimulation of either the primary motor cortex or the dorsal premotor cortex to reduce MEP amplitudes significantly (Chouinard et al., 2003). We expected also to see a gradual return of MEP amplitudes compared with baseline measurements $20 \mathrm{~min}$ after rTMS (Chouinard et al., 2003).

We used a four-step procedure to place the TMS coil over the primary motor and dorsal premotor cortices (Paus et al., 1997). First, we transformed the subject's MRI into standardized space (Talairach and Tournoux, 1988; Collins et al., 1994). Second, we derived probabilistic locations for the primary motor $(X=-31, Y=-22, Z=52)$ and dorsal premotor $(X=-21, Y=-2, Z=52)$ cortices using information gained in previous brain imaging studies (Paus et al., 1998; Chouinard et al., 2003). Third, we transformed the probabilistic locations to the subject's brain coordinate space. Fourth, we used frameless stereotaxy to position the TMS coil over the probabilistic locations marked on the subject's MRI (Brainsight software, Rogue Research, Montreal, Quebec, Canada; Polaris System, Northern Digital, Waterloo, Ontario, Canada). In the case of the primary motor cortex, we made additional adjustments in coil positioning to where stimulation resulted in the maximum MEP amplitude.

For single-pulse TMS, we applied 20 single pulses of stimulation every $5 \pm 1 \mathrm{~s}$ at a suprathreshold intensity of $120 \%$ of the resting motor threshold. For rTMS, we applied $15 \mathrm{~min}$ of $1 \mathrm{~Hz}$ repetitive stimulation at a subthreshold intensity of $90 \%$ of the resting motor threshold in three 5 min blocks, each block separated by $1 \mathrm{~min}$, to minimize overheating of the stimulating coil. Subthreshold intensities allow for more focal stimulation by narrowing the magnetic field produced by the coil, thus enabling better spatial resolution for examining changes between different cortical structures (Pascual-Leone et al., 1993). We held the coil in the same orientation when stimulating both the primary motor and dorsal premotor cortices. We oriented the coil tangentially to the scalp with the short axis of the figure-of-eight coil angled $45^{\circ}$ relative to the interhemispheric fissure and approximately perpendicular to the central sulcus. For both primary motor and dorsal premotor stimulation, the resulting induced current in the brain flowed in a posterior-to-anterior and lateral-to-medial direction.

Verification of coil positions. We derived projected coil trajectories from the center of the figure-of-eight coil using the Brainsight software (see previous section) as an estimation of where stimulation took place. After placing the coil over the sites of stimulation, we saved the projected coil trajectories in the subject's brain coordinate space. We then marked on the subject's MRI where this trajectory passed in the same perpendicular plane, or parallel plane to the coil, as the site we intended to target. We then transformed these coordinates from voxel space to native space using the software Register (Montreal Neurological Institute, Montreal, Quebec, Canada) and then to standardized space. Projected coil trajectories for the primary motor cortex revealed minimal overlap with those for the dorsal premotor cortex (see Fig. 2A,C). Projected coil trajectories for the dorsal premotor cortex generally passed in the rostral dorsal premotor cortex as established by Piccard and Strick (2000). Projected coil trajectories for the primary motor cortex showed greater variability. This is likely because we made adjustments in coil positioning to target where stimulation resulted in the maximum MEP amplitude; previous studies have reported that this location can vary among individuals (Classen et al., 1998).

Analyses for motor-evoked potentials. For both the no-cue and cue experiments, we evaluated the effects of repetitive stimulation on motor excitability by ANOVA using time and site of stimulation as withinsubject factors. We used Tukey's honestly significant difference (HSD) pair-wise comparison tests, which corrected for multiple comparisons, to examine additional significant effects. We also used paired $t$ tests to compare resting motor thresholds values acquired during sessions with repetitive stimulation over the primary motor cortex with those acquired during sessions with repetitive stimulation over the dorsal premotor cortex.

Analyses for precision grip. Using Matlab, we measured the rates in grip force for each but the first trial and then calculated the means for each of the different four conditions for each block. For measuring the rates in grip force, we divided the magnitude of the peak force by the time difference between the peak grip force and the first increase in grip-force signal. For the statistical analyses of rates in grip force, we performed an ANOVA that examined the effects of repetitive stimulation on performance in each of the two experiments. For this ANOVA, we used switching (no switch vs switch), weight (light vs heavy), block (20-12 min before rTMS vs 10 -2 min before rTMS vs 12-20 min after rTMS vs 22-30 min after rTMS), and site of stimulation (primary motor vs dorsal premotor) as within-subject factors.

We also performed additional ANOVAs on both the rates in load force and the load force time (time of peak force - time of first increase in signal) in cases in which the rates in grip force changed during an experiment. We calculated the rates in load force the same way as we calculated the rates in grip force. For these ANOVAs, we used switching (no switch vs switch), weight (light vs heavy), and block (20-12 min before rTMS vs 10-2 min before rTMS vs $12-20 \mathrm{~min}$ after rTMS vs 22-30 min after rTMS) as within-subject factors. We performed simple effect tests and Tukey's HSD pair-wise comparison tests, which corrected for multiple comparisons, to examine additional significant interactions.

\section{Results}

\section{Resting motor thresholds}

Paired $t$ tests on the resting motor thresholds revealed no difference between sessions in both the no-cue $\left(t_{(7)}=0.39 ; p=0.71\right)$ and cue $\left(t_{(7)}=0.24 ; p=0.82\right)$ experiments.

\section{No-cue experiment}

Effects of repetitive stimulation on motor excitability

An ANOVA on the MEP amplitudes revealed an effect of time $\left(F_{(6,42)}=4.76 ; p<0.001\right)$, no effect of site of stimulation $\left(F_{(1,7)}=\right.$ 
$0.26 ; p=0.63)$, and no time $\times$ site of stimulation interaction $\left(F_{(6,42)}=1.52 ; p=\right.$ $0.20)$. These results demonstrate that changes in MEP amplitudes did not differ when we applied repetitive stimulation over the primary motor cortex compared with the dorsal premotor cortex (Fig. $2 B$ ). We performed Tukey's HSD tests to examine further the effect of time and found reductions in MEP amplitudes $0-2$ min after rTMS compared with 22-20 min before rTMS $(p<0.05), 12-10$ min before rTMS $(p<0.01), 2-0$ min before rTMS $(p<$ $0.05), 20-22 \mathrm{~min}$ after rTMS $(p<0.01)$, and 30-32 min after rTMS $(p<0.01)$.

\section{Effects of repetitive stimulation on} grip forces

An ANOVA on the rates in grip force revealed a significant switching $X$ weight $X$ block $X$ site of stimulation interaction $\left(F_{(3,21)}=3.42 ; p<0.05\right)$.

Before repetitive stimulation, subjects applied rates in force that reflected the scaling of forces for a previous weight (Fig. $3 A-C$ ). In the no switch trials (i.e., when subjects lifted the same weight as in the previous lift), subjects applied faster rates in grip force when they lifted the heavy weight compared with the light weight (heavy-after-heavy > light-after-light). When changes in weight occurred between lifts, however, effects of switching were present. In the switch trials (i.e., when subjects lifted a different weight than in the previous lift), the rates in grip force increased after the weight became lighter (light-after-heavy $>$ light-afterlight) and decreased after the weight became heavier (heavy-after-light $<$ heavyafter-heavy). These effects of switching indicate that subjects scaled their grip forces based on the previous weight. Repetitive stimulation over the dorsal premotor cortex had no effect on the rates in grip force (Fig. 3B).

Repetitive stimulation over the primary motor cortex disrupted the production of distinct rates in grip force (Fig. $3 A, D)$. In the no switch trials, the rates in grip force at 12-20 min after rTMS did not differ when subjects lifted the heavy weight compared with the light weight (heavy-after-heavy $\approx$ light-after-light). In the switch trials, the rates in grip force at 12-20 min after rTMS did not increase after the weight became lighter (light-after-heavy $\approx$ light-afterlight) and did not decrease after the weight became heavier (heavy-after-light $\approx$ heavy-after-heavy). A power analysis revealed that a sample size of 37 subjects would be necessary to reject the null hypothesis that no effects of switching occurred $(\alpha=0.05)$. At $22-30 \mathrm{~min}$ after rTMS, the rates in grip force were similar to those before repetitive stimulation. These results indi-
No-Cue Experiment

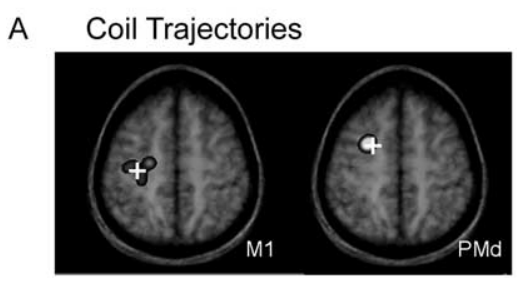

B MEP Amplitudes

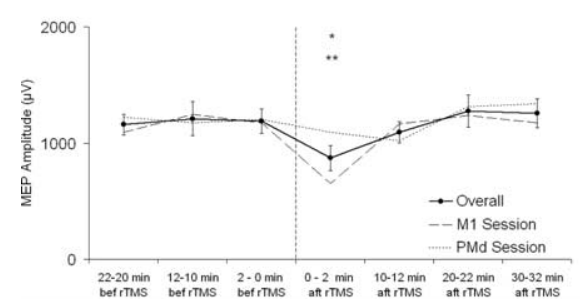

\section{Cue Experiment}

\section{Coil Trajectories}

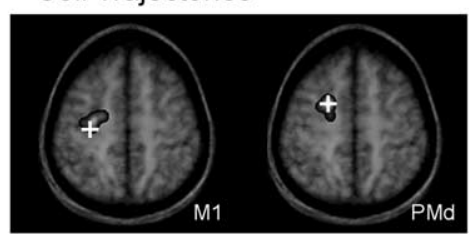

D MEP Amplitudes

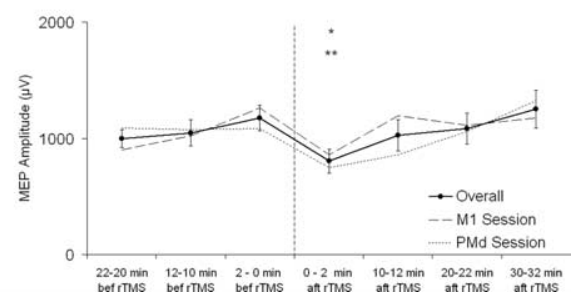

Figure 2. MEP amplitudes. $A, C$, Superimposed on magnetic resonance images are projected coil trajectories that indicate estimated locations for induced currents in the brain during repetitive stimulation over the primary motor (M1) and dorsal premotor (PMd) cortices. The brightness of these superimpositions reflects the probability of the coil trajectories. Crosses represent their probabilistic locations. $\boldsymbol{B}, \boldsymbol{D}$, Overall mean \pm SEM MEP amplitudes and MEP amplitudes in the primary motor and dorsal premotor sessions are shown. Asterisks denote significant differences for overall MEP amplitudes (no-cue experiment: ${ }^{*} p<0.05$ vs $22-20$ min before rTMS, $2-0$ min before rTMS; ${ }^{* *} p<0.01$ vs $12-10$ min before rTMS, $20-22$ min after rTMS, $30-32$ min after rTMS; cue experiment: ${ }^{*} p<0.05$ vs $2-0$ min before rTMS; ${ }^{* *} p<0.01$ vs $30-32$ min after rTMS).

\section{Grip Forces in No-Cue Experiment}

B PMd Session

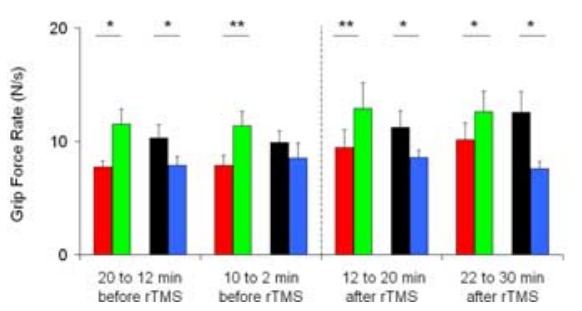

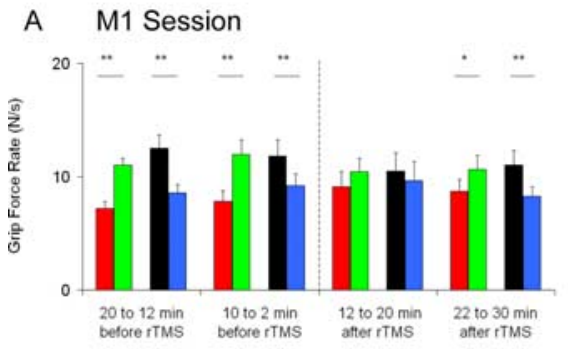

C

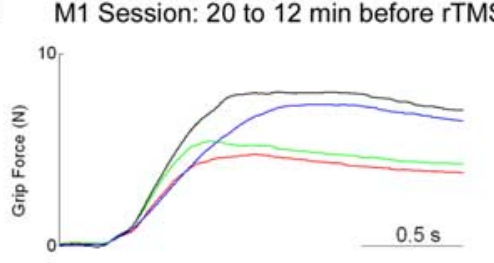

D M1 Session: 12 to 20 min after rTMS

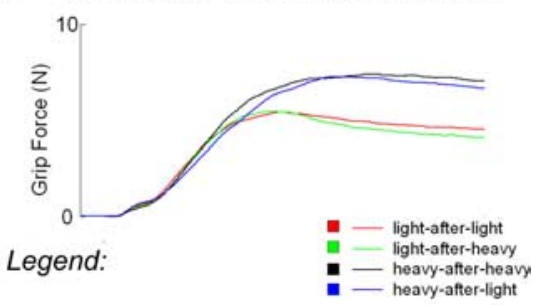

Figure 3. Grip forces in the no-cue experiment. $A$ represents means \pm SEM for the rates in grip force before and after repetitive stimulation over the primary motor cortex (M1). $\boldsymbol{B}$ represents means \pm SEM for the rates in grip force before and after repetitive stimulation over the dorsal premotor cortex (PMd). C represents the overall average traces for grip forces 20-12 min before repetitive stimulation over M1. D represents the overall average traces for grip forces $12-20 \mathrm{~min}$ after repetitive stimulation over M1. Asterisks denote significant differences between switch conditions $\left({ }^{*} p \leq 0.05 ;{ }^{* *} p<0.01\right)$.

cate that repetitive stimulation over the primary motor cortex temporarily disrupted the subjects' ability to apply distinct rates in grip force when lifting different weights and to scale forces based on the previous weight.

\section{Effects of repetitive stimulation on load forces}

An ANOVA on the rates in load force showed a switching $\times$ weight $X$ block interaction in the session with repetitive stimu- 
Load Forces in No-Cue Experiment

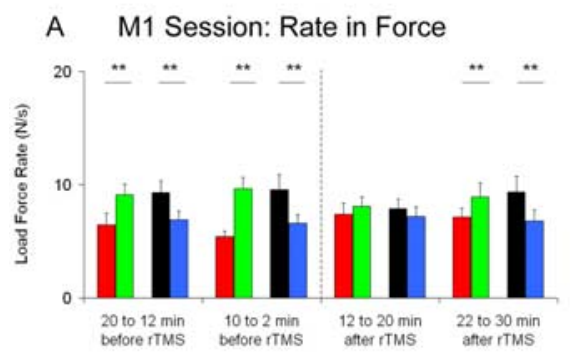

\section{B M1 Session: Time in Force}

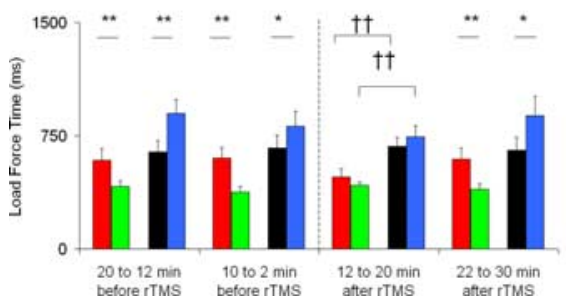

D

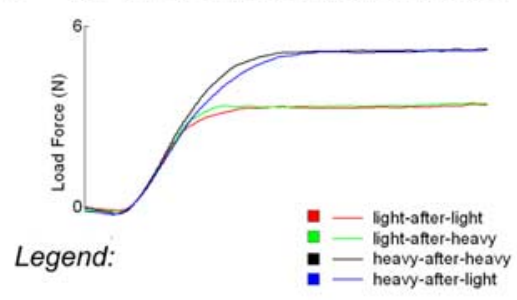

Figure 4. Load forces in the no-cue experiment. $\boldsymbol{A}$ represents means \pm SEM for the rates in load force before and after repetitive stimulation over the primary motor cortex (M1). $\boldsymbol{B}$ represents means \pm SEM for the load-force times before and after repetitive stimulation over $M 1$. Crepresents the overall average traces for load forces $20-12$ min before repetitive stimulation over M1. D represents the overall average traces for load forces $12-20 \mathrm{~min}$ after repetitive stimulation over M1. Asterisks denote significant differences between switch conditions $\left({ }^{*} p \leq 0.05\right.$; $\left.{ }^{* *} p<0.01\right)$. Daggers denote significant differences between weight conditions at post- $1\left({ }^{\dagger \dagger} p<0.01\right)$.

lation over the primary motor cortex $\left(F_{(3,21)}=7.26 ; p<0.005\right)$. Additional examination of this interaction reveals similar effects as those observed for the rates in grip force (Fig. $4 A, C, D$ ). An ANOVA performed on the load force times (Fig. $4 B$ ) also showed a significant switching $X$ weight $\times$ block interaction in the session with repetitive stimulation over the primary motor cortex $\left(F_{(3,21)}=5.98 ; p<0.005\right)$.

Before repetitive stimulation, the load-force times did not differ in the no-switch trials when subjects lifted the heavy weight compared with the light weight (heavy-after-heavy $\approx$ light-afterlight). Effects of switching, however, were present when changes in weight occurred between lifts. In the switch trials, the loadforce times decreased after the weight became lighter (light-afterheavy $<$ light-after-light) and increased after the weight became heavier (heavy-after-light $>$ heavy-after-heavy).

Repetitive stimulation over the primary motor cortex resulted in distinct load-force times for the two different weights (Fig. $4 B, D)$. In the no-switch trials, the load-force times at 12-20 min after rTMS were longer when subjects lifted the heavy weight compared with the light weight (heavy-after-heavy $>$ light-afterlight). In the switch trials, the load-force times at 12-20 min after rTMS did not decrease after the weight became lighter (lightafter-heavy $\approx$ light-after-light) nor did they increase after the weight became heavier (heavy-after-light $\approx$ heavy-after-heavy). At 22-30 min after rTMS, the load-force times were similar to those before repetitive stimulation. These results suggest that although subjects at 12-20 min after rTMS applied similar rates in force in all four weight $\times$ switching conditions, the time to scale load forces prolonged for the heavy weight compared with the light weight.

\section{The cue experiment}

Effects of repetitive stimulation on motor excitability

An ANOVA on the MEP amplitudes showed an effect of time $\left(F_{(6,42)}=3.12 ; p<0.05\right)$, no effect of site of stimulation $\left(F_{(1,7)}=\right.$
$0.10 ; p=0.76)$, and no interaction of time $\times$ site of stimulation $\left(F_{(6,42)}=1.85\right.$; $p=0.11)$. These results demonstrate that changes in MEP amplitudes did not differ when we applied repetitive stimulation over the primary motor cortex compared with the dorsal premotor cortex. We performed Tukey's HSD tests to examine further the effect of time (Fig. 2D) and found significant reductions in MEP amplitudes 0-2 min after rTMS compared with 2-0 min before rTMS $(p<0.05)$ and 30-32 min after rTMS $(p<0.01)$.

\section{Effects of repetitive stimulation on grip forces}

An ANOVA on the rates in grip force showed a significant switching $X$ weight $\times$ block $\times$ site of stimulation interaction $\left(F_{(3,21)}=5.83 ; p<0.005\right)$.

Before repetitive stimulation, subjects in the cue experiment could use arbitrary color cues to scale rates in grip force for a current weight (Fig. 5A-C). In the noswitch trials, subjects applied faster rates in grip force when they lifted the heavy weight compared with the light weight (heavy-after-heavy $>$ light-after-light). In the switch trials, unlike the no-cue experiment, the rates in grip force did not differ after the weight became either lighter (light-after-heavy $\approx$ light-after-light) or heavier (heavy-after-light $\approx$ heavy-after-heavy). The lack of any switching effect indicates that subjects could use arbitrary color cues to scale for forces based on the current weight, even during trials after a switch in weight occurred between lifts. Repetitive stimulation over the primary motor cortex had no effect on the rates in grip force (Fig. 5A).

Repetitive stimulation over the dorsal premotor cortex resulted in the scaling of rates in grip force for a previous lift (Fig. $5 B, D)$. In the switch trials, the rates in grip force at 12-20 min after rTMS increased after the weight became lighter (light-afterheavy $>$ light-after-light) and decreased after the weight became heavier (heavy-after-light $<$ heavy-after-heavy). At 22-30 min after rTMS, the rates in grip force were similar to those before repetitive stimulation. Direct comparisons between the lightafter-heavy conditions confirm that subjects scaled their forces for a previous weight at 12-20 min after rTMS; the rates in grip force were faster when subjects lifted the light weight after the heavy weight at 12-20 min after rTMS compared with both before and 22-30 min after rTMS.

\section{Effects of repetitive stimulation on load forces}

An ANOVA performed on the rates in load force in the session with repetitive stimulation over the dorsal premotor cortex revealed a switching $\times$ weight $\times$ block interaction $\left(F_{(3,21)}=3.91\right.$; $p<0.05)$. These results are similar to those observed for the rates in grip force (Fig. $6 A, C, D$ ). The same ANOVA on the load-force times (Fig. $6 B$ ) also revealed a switching $\times$ weight $\times$ block interaction $\left(F_{(3,21)}=10.93 ; p<0.001\right)$.

Before repetitive stimulation, subjects applied longer loadforce times for the heavy weight compared with the light weight (heavy-after-heavy $>$ light-after-light). Effects of switching, however, were not present when changes in weight occurred between lifts. In the switch trials, the load-force times did not differ 
after the weight became lighter (light-after-heavy $\approx$ light-after-light) nor did they differ after the weight became heavier (heavy-after-light $\approx$ heavy-after-heavy).

After repetitive stimulation over the dorsal premotor cortex, subjects applied loadforce times that reflected the scaling of forces for a previous weight (Fig. $6 B, D$ ). In the noswitch trials, the load-force times at 12-20 min after rTMS did not differ when subjects lifted the heavy weight compared with the light weight (heavy-after-heavy $\approx$ lightafter-light). In the switch trials, the loadforce times at 12-20 min after rTMS decreased after the weight became lighter (light-after-heavy $<$ heavy-after-heavy) and increased after the weight became heavier (heavy-after-light $>$ heavy-after-heavy). At 22-30 min after rTMS, the load-force times were similar to those before repetitive stimulation.

\section{Discussion}

Our results demonstrate that lowfrequency repetitive stimulation applied over the primary motor and dorsal premotor cortices influenced differentially the anticipatory scaling of forces. When applied over the primary motor cortex, repetitive stimulation disrupted the scaling of forces based on information acquired during a previous lift. In contrast, when applied over the dorsal premotor cortex, repetitive stimulation disrupted the scaling of forces based on arbitrary color cues. Together, these findings indicate that during the lifting of different weights, the primary motor cortex scales forces based on information acquired during a previous lift and the dorsal premotor cortex scales forces based on arbitrary visual cues.

\section{Methodological issues}

Reductions in motor excitability occurred immediately after repetitive stimulation compared with a $\sim 10$ min delay observed in our previous TMS/positron emission tomography study (Chouinard et al., 2003). We speculate that the performance of precision grip before repetitive stimulation might have had a "priming" effect on motor excitability (Iyer et al., 2003). Note that changes in MEPs related modestly to changes in the precision grip. Thus, the MEP data provide only a verification of the effectiveness of rTMS over the two sites (Chouinard et al., 2003).

Our study did not examine the scaling of forces based on information about the three-dimensional (3-D) characteristics of objects. Both shape and size can influence the anticipatory scaling of forces that are applied during the lifting of small objects (Gordon et al., 1991a,b; Jenmalm and Johansson, 1997, 2000; Goodwin et al., 1998; Flanagan et al., 2001). The dorsal premotor cortex selects movements based mainly on learned associations as

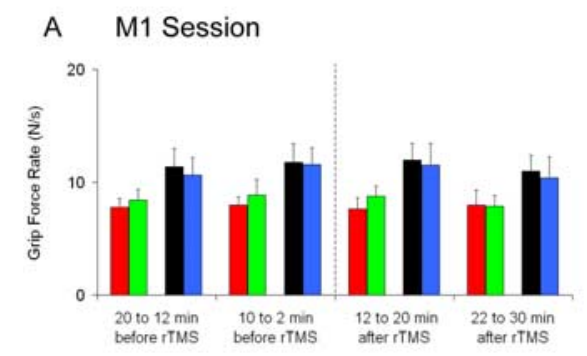

C PMd Session: 20 to 12 min before rTMS

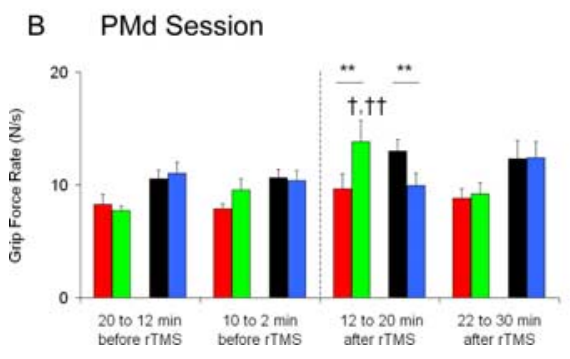

D PMd Session: 12 to 20 min after rTMS

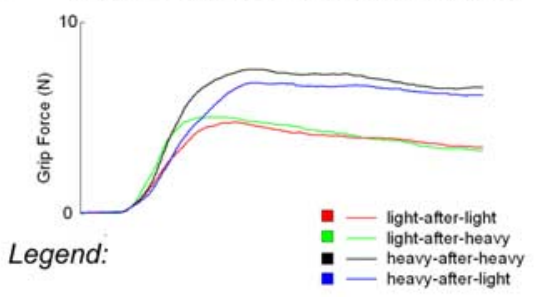

Figure 5. Grip forces in the cue experiment. $\boldsymbol{A}$ represents means \pm SEM for the rates in grip force before and after repetitive stimulation over the primary motor cortex (M1). $\boldsymbol{B}$ represents means \pm SEM for the rates in grip force before and after repetitive stimulation over the dorsal premotor cortex (PMd). C represents the overall average traces for grip forces 20 -12 min before repetitive stimulation over PMd. $D$ represents the overall average traces for grip forces $12-20$ min after repetitive stimulation over PMd. Asterisks denote significant differences between switch conditions $\left({ }^{*} p \leq 0.05 ;{ }^{* *} p<0.01\right)$. Daggers denote significant differences between block conditions ( ${ }^{\dagger} p<0.05$ vs pre- $2 ;{ }^{\dagger+} p<0.01$ vs pre- 1 and post-2).

Load Forces in Cue Experiment

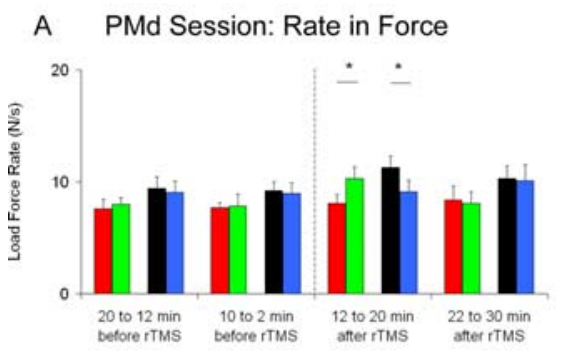

B PMd Session: Time in Force

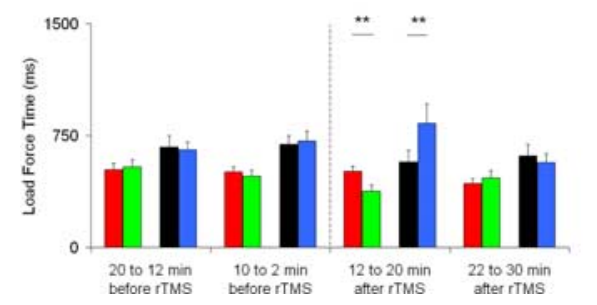

Figure 6. Load forces in the cue experiment. $A$ represents means \pm SEM for the rates in load force before and after repetitive stimulation over the dorsal premotor cortex (PMd). $\boldsymbol{B}$ represents means SEM for the load-force times before and after repetitive stimulation over PMd. C represents the overall average traces for load forces $20-12 \mathrm{~min}$ before repetitive stimulation over PMd. $\boldsymbol{D}$ represents the overall average traces for load forces $12-20 \mathrm{~min}$ after repetitive stimulation over PMd. Asterisks denote significant differences between switch conditions $\left({ }^{*} p \leq 0.05 ;{ }^{* *} p<0.01\right)$.

opposed to the more pragmatic visual and somatosensory analyses of shape and size (Geyer et al., 2000). In contrast, the ventral premotor cortex receives both visual and somatosensory information about the 3-D characteristics of objects from the anterior intraparietal area (Murata et al., 1997; Rizzolatti et al., 1998).

Our study also did not examine the actual learning of associations. Subjects in the cue experiment learned to associate quickly arbitrary color cues with weight and we found no differences in 
performance during the two blocks before repetitive stimulation. Previous studies demonstrate that subjects can learn new associations as quickly as by the second trial in situations when the same weight has changed in physical size from bigger to smaller or smaller to bigger (Gordon et al., 1991b; Flanagan et al., 2001). To examine the actual learning of associations, we would have had to resort to a task that involves more associations and is more taxing than just two arbitrary cues and two corresponding motor outputs. Such a task would have to match in difficulty as the ones used in studies conducted by Petrides (1985b, 1997) in which six or nine different colored lights each cued subjects to perform a different hand gesture.

\section{Primary motor cortex and anticipatory scaling of forces}

As demonstrated in this study and in previous studies, when the weight of an object changes unexpectedly without any visible changes in appearance, people will generate inappropriate forces on the first lift and quite accurate forces on the second lift (Johansson and Westling, 1988; Gordon et al., 1993; Fellows et al., 1998). Measurements acquired in the no-cue experiment demonstrate that: (1) when switches in weight occurred between lifts, subjects scaled rates in force appropriate for a previous weight and not for a current weight, (2) both the production of distinct rates in force and the scaling of forces for a previous weight diminished 12-20 min after repetitive stimulation applied over the primary motor cortex and reemerged $22-30 \mathrm{~min}$ after repetitive stimulation, and (3) repetitive stimulation applied over the dorsal premotor cortex affected neither the production of distinct rates in force nor the scaling of forces for a previous weight.

In the no-cue experiment, repetitive stimulation applied over the primary motor cortex temporarily disrupted the subjects' ability to apply distinct rates in force when lifting different weights and to scale forces based on the previous weight. Additional analyses revealed that although subjects applied similar rates in force in all four weight $\times$ switching conditions, the loadforce times prolonged for the heavy weight compared with the light weight. This additional finding suggests that subjects applied similar rates in load force until a sufficient vertical force was reached to overcome gravity.

It is important to note that in the cue experiment, repetitive stimulation over the primary motor cortex had no effect on the subjects' ability to scale forces for a current weight. This is likely because the arbitrary color cues provided subjects with information about what weight they had to lift, and the subjects were able to use this information to scale for differences in weight. Thus, the observed effects induced by repetitive stimulation over the primary motor cortex do not appear to be at the level of motor execution, but rather at the level of processing motor information associated with a recent experience. Indeed, a number of TMS studies have reported a similar lack of effects on motor execution despite reductions in motor excitability after low-frequency repetitive stimulation over the primary motor cortex (Chen et al., 1997; Muellbacher et al., 2000, 2002).

We speculate that the primary motor cortex can form memory traces associated with a recent experience. Cell recording studies in the monkey reveal that separate populations of primary motor neurons can process information related to motor function (Georgopoulos et al., 1982; Zhang et al., 1997; Wise et al., 1998; Li et al., 2001), including a population of memory neurons that stores information related to an experience beyond its duration. Li et al. (2001) examined activity in primary motor neurons before, during, and after motor adaptation. Their results revealed that a subset of neurons, which they called memory neurons, changed their firing properties as monkeys learned to perform forelimb movements in a force field. Once the force field was turned off, the firing properties of the memory neurons remained altered, and monkeys in turn produced inappropriate forelimb movements. A recent TMS study also demonstrated that repetitive stimulation over the primary motor cortex can disrupt adaptation in a similar force-field task (Cothros et al., 2004).

\section{Dorsal premotor cortex and anticipatory scaling of forces}

Measurements acquired in the cue experiment demonstrate that: (1) when switches in weight occurred between lifts, subjects could use arbitrary color cues to scale rates in force for a current weight, (2) the ability to use arbitrary color cues to scale rates in force for a current weight diminished 12-20 min after repetitive stimulation applied over the dorsal premotor cortex and reemerged 22-30 min repetitive stimulation, and (3) repetitive stimulation applied over the primary motor cortex did not affect the production of scaling of forces based on the arbitrary color cues.

Contrary to the first observation, Cole and Rotella (2002) found that subjects applied grip forces from a previous lift even in cases when they lifted different colored objects in which color informed them about texture. We speculate that the reason for this discrepancy is that subjects in Cole and Rotella's study had to extract and dissociate color from other visual characteristics (e.g., shape, size) that the brain could have associated with properties of the object during the previous lift. This differs from our study in which we presented arbitrary color cues on a computer screen.

The scaling of forces for a previous weight is associated with somatosensory information related to errors made during weight prediction (Johansson and Westling, 1988). An alternative explanation for our results could be that repetitive stimulation applied over the dorsal premotor cortex enhanced the use of somatosensory information from a previous trial in a manner that would drive subjects to ignore the cues and scale forces based on the previous weight. We argue against this possibility for two reasons. First, repetitive stimulation over the dorsal premotor cortex in the no-cue experiment had no effect on the manner with which subjects scaled forces for a previous weight. Second, cell recording studies in the monkey demonstrate that the dorsal premotor cortex contains only a few neurons that use somatosensory information to control for corrective forces during the precision grip (Boudreau et al., 2001).

Thus, the observed effects induced by repetitive stimulation over the dorsal premotor cortex appear to be at the level of coupling arbitrary visual cues and motor output. Indeed, cell recording studies in the monkey reveal that a number of dorsal premotor neurons increase their discharge activity after the presentation of an arbitrary visual cue that represents a learned association for a particular motor response compared with the presentation of a directional cue indicating a particular motor response (Kurata and Wise, 1988; Mitz et al., 1991; Kurata and Hoffman, 1994). GABA ${ }_{\mathrm{A}}$-agonist muscimol injections in the dorsal premotor cortex diminish the monkey's ability to select a correct response based on an arbitrary visual cue (Kurata and Hoffman, 1994). Petrides (1982, 1985a), as well as Halsband and Passingham $(1982,1985)$, have shown that the removal of the dorsal premotor cortex disrupts the ability to use arbitrary visual cues to make or withhold particular movements. Our current observations, together with these findings, reinforce the notion that the premotor cortex is critical for implementing associations between visual cues and motor responses. 


\section{References}

Boudreau MJ, Brochier T, Pare M, Smith AM (2001) Activity in ventral and dorsal premotor cortex in response to predictable force-pulse perturbations in a precision grip task. J Neurophysiol 86:1067-1078.

Chen R, Classen J, Gerloff C, Celnik P, Wassermann EM, Hallett M, Cohen LG (1997) Depression of motor cortex excitability by low-frequency transcranial magnetic stimulation. Neurology 48:1398-1403.

Chouinard PA, Van Der Werf YD, Leonard G, Paus T (2003) Modulating neural networks with transcranial magnetic stimulation applied over the dorsal premotor and primary motor cortices. J Neurophysiol 90:1071-1083.

Classen J, Knorr U, Werhahn KJ, Schlaug G, Kunesch E, Cohen LG, Seitz RJ, Benecke R (1998) Multimodal output mapping of human central motor representation on different spatial scales. J Physiol (Lond) 512:163-179.

Cohen LG, Roth BJ, Nilsson J, Dang N, Panizza M, Bandinelli S, Friauf W, Hallett M (1990) Effects of coil design on delivery of focal magnetic stimulation. Technical considerations. Electroencephalogr Clin Neurophysiol 75:350-357.

Cole KJ, Rotella DL (2002) Old age impairs the use of arbitrary visual cues for predictive control of fingertip forces during grasp. Exp Brain Res 143:35-41.

Collins DL, Neelin P, Peters TM, Evans AC (1994) Automatic 3D intersubject registration of MR volumetric data in standardized Talairach space. J Comput Assist Tomogr 18:192-205.

Cothros N, Dickie EW, Köhler S, Gribble PL (2004) Role of primary motor cortex in consolidation and interference during motor learning. Soc Neurosci Abstr 30:871.15

Crovitz HF, Zener K (1965) A group for assessing hand and eye-dominance. Am J Psychol 75:271-276.

Fellows SJ, Noth J, Schwarz M (1998) Precision grip and Parkinson's disease. Brain 121:1771-1784.

Flanagan JR, King S, Wolpert DM, Johansson RS (2001) Sensorimotor prediction and memory in object manipulation. Can J Exp Psychol 55:87-95.

Georgopoulos AP, Kalaska JF, Caminiti R, Massey JT (1982) On the relations between the direction of two-dimensional arm movements and cell discharge in primate motor cortex. J Neurosci 2:1527-1537.

Geyer S, Matelli M, Luppino G, Zilles K (2000) Functional neuroanatomy of the primate isocortical motor system. Anat Embryol (Berl) 202:443-474.

Goodwin AW, Jenmalm P, Johansson RS (1998) Control of grip force when tilting objects: effect of curvature of grasped surfaces and applied tangential torque. J Neurosci 18:10724-10734.

Gordon AM, Forssberg H, Johansson RS, Westling G (1991a) Visual size cues in the programming of manipulative forces during precision grip. Exp Brain Res 83:477-482.

Gordon AM, Forssberg H, Johansson RS, Westling G (1991b) The integration of haptically acquired size information in the programming of precision grip. Exp Brain Res 83:483-488.

Gordon AM, Westling G, Cole KJ, Johansson RS (1993) Memory representations underlying motor commands used during manipulation of common and novel objects. J Neurophysiol 69:1789-1796.

Halsband U, Passingham R (1982) The role of premotor and parietal cortex in the direction of action. Brain Res 240:368-372.

Halsband U, Passingham RE (1985) Premotor cortex and the conditions for movement in monkeys (Macaca fascicularis). Behav Brain Res 18:269-277.

Iyer MB, Schleper N, Wassermann EM (2003) Priming stimulation enhances the depressant effect of low-frequency repetitive transcranial magnetic stimulation. J Neurosci 23:10867-10872.

Jenmalm P, Johansson RS (1997) Visual and somatosensory information about object shape control manipulative fingertip forces. J Neurosci 17:4486-4499.

Jenmalm P, Dahlstedt S, Johansson RS (2000) Visual and tactile information about object-curvature control fingertip forces and grasp kinematics in human dexterous manipulation. J Neurophysiol 84:2984-2997.

Johansson RS (1996) Sensory and memory information in the control of dexterous manipulation. In: Neural basis of motor behaviour (Lacquaniti F, Viviani P, eds), pp 205-260. Dordrecht, The Netherlands: Kluwer Academic.

Johansson RS, Westling G (1984) Roles of glabrous skin receptors and sensorimotor memory in automatic control of precision grip when lifting rougher or more slippery objects. Exp Brain Res 56:550-564.

Johansson RS, Westling G (1988) Coordinated isometric muscle commands adequately and erroneously programmed for the weight during lifting task with precision grip. Exp Brain Res 71:59-71.
Kurata K, Hoffman DS (1994) Differential effects of muscimol microinjection into dorsal and ventral aspects of the premotor cortex of monkeys. J Neurophysiol 71:1151-1164.

Kurata K, Wise SP (1988) Premotor cortex of rhesus monkeys: set-related activity during two conditional motor tasks. Exp Brain Res 69:327-343.

Li CS, Padoa-Schioppa C, Bizzi E (2001) Neuronal correlates of motor performance and motor learning in the primary motor cortex of monkeys adapting to an external force field. Neuron 30:593-607.

Maccabee PJ, Eberle L, Amassian VE, Cracco RQ, Rudell A, Jayachandra M (1990) Spatial distribution of the electric field induced in volume by round and figure " 8 " magnetic coils: relevance to activation of sensory nerve fibers. Electroencephalogr Clin Neurophysiol 76:131-141.

Mitz AR, Godschalk M, Wise SP (1991) Learning-dependent neuronal activity in the premotor cortex: activity during the acquisition of conditional motor associations. J Neurosci 11:1855-1872.

Muellbacher W, Ziemann U, Boroojerdi B, Hallett M (2000) Effects of lowfrequency transcranial magnetic stimulation on motor excitability and basic motor behavior. Clin Neurophysiol 111:1002-1007.

Muellbacher W, Ziemann U, Wissel J, Dang N, Kofler M, Facchini S, Boroojerdi B, Poewe W, Hallett M (2002) Early consolidation in human primary motor cortex. Nature 415:640-644.

Munchau A, Bloem BR, Irlbacher K, Trimble MR, Rothwell JC (2002) Functional connectivity of human premotor and motor cortex explored with repetitive transcranial magnetic stimulation. J Neurosci 22:554-561.

Murata A, Fadiga L, Fogassi L, Gallese V, Raos V, Rizzolatti G (1997) Object representation in the ventral premotor cortex (area F5) of the monkey. J Neurophysiol 78:2226-2230.

Pascual-Leone A, Houser CM, Reese K, Shotland LI, Grafman J, Sato S, VallsSole J, Brasil-Neto JP, Wassermann EM, Cohen LG (1993) Safety of rapid-rate transcranial magnetic stimulation in normal volunteers. Electroencephalogr Clin Neurophysiol 89:120-130.

Paus T, Jech R, Thompson CJ, Comeau R, Peters T, Evans AC (1997) Transcranial magnetic stimulation during positron emission tomography: a new method for studying connectivity of the human cerebral cortex. J Neurosci 17:3178-3184.

Paus T, Jech R, Thompson CJ, Comeau R, Peters T, Evans AC (1998) Dosedependent reduction of cerebral blood flow during rapid-rate transcranial magnetic stimulation of the human sensorimotor cortex. J Neurophysiol 79:1102-1107.

Petrides M (1982) Motor conditional associative-learning after selective prefrontal lesions in the monkey. Behav Brain Res 5:407-413.

Petrides M (1985a) Deficits in non-spatial conditional associative learning after periarcuate lesions in the monkey. Behav Brain Res 16:95-101.

Petrides M (1985b) Deficits on conditional associative-learning tasks after frontal- and temporal-lobe lesions in man. Neuropsychologia 23:601-614.

Petrides M (1997) Visuo-motor conditional associative learning after frontal and temporal lesions in the human brain. Neuropsychologia 35:989-997.

Piccard N, Strick PL (2000) Imaging the premotor areas. Curr Opin Neurobiol 11:663-672, 2000.

Rizzolatti G, Luppino G, Matelli M (1998) The organization of the cortical motor system: new concepts. Electroencephalogr Clin Neurophysiol 106:283-296.

Talairach J, Tournoux P (1988) Co-planar stereotactic atlas of the human brain: 3-dimensional proportional system: an approach to cerebral imaging. Stuttgard, Germany: Thieme.

Touge T, Gerschlager W, Brown P, Rothwell JC (2001) Are the after-effects of low-frequency rTMS on motor cortex excitability due to changes in the efficacy of cortical synapses? Clin Neurophysiol 112:2138-2145.

Wassermann EM, Wang B, Zeffiro TA, Sadato N, Pascual-Leone A, Toro C, Hallett M (1996) Locating the motor cortex on the MRI with transcranial magnetic stimulation and PET. NeuroImage 3:1-9.

Wise SP, Murray EA (2000) Arbitrary associations between antecedents and actions. Trends Neurosci 23:271-276.

Wise SP, Moody SL, Blomstrom KJ, Mitz AR (1998) Changes in motor cortical activity during visuomotor adaptation. Exp Brain Res 121:285-299.

Wolpert DM, Flanagan JR (2001) Motor prediction. Curr Biol 11:R729-R732.

Zhang J, Riehle A, Requin J, Kornblum S (1997) Dynamics of single neuron activity in monkey primary motor cortex related to sensorimotor transformation. J Neurosci 17:2227-2246. 